\title{
A Fuzzy Model for the Multiobjective Emergency Facility Location Problem with $A$-Distance
}

\author{
T. Uno*, H. Katagiri and K. Kato \\ Department of Artificial Complex Systems Engineering, Graduate School of Engineering, Hiroshima University, \\ Higashihiroshima, Hiroshima, Japan
}

\begin{abstract}
This paper proposes a new multiobjective location problem for emergency facilities, e.g. ambulance service stations and fire stations. In the problem, one of the objectives is to minimize the maximal distance of the paths from the located emergency facilities to hospitals via accidents. The other is to maximize the frequency of accidents that the emergency facilities can respond quickly. An interactive fuzzy satisficing method with particle swarm optimization (PSO) method is proposed for finding a satisficing location for the problem. Computational results illustrate the method with numerical examples of the multiobjective location problem.
\end{abstract}

\section{INTRODUCTION}

In this paper, we consider a new emergency facility location problem (EFLP), such that ambulance service stations [1-3], fire stations [1, 4, 5], etc. Matsutomi and Ishii [2] considered EFLPs with the situation that if an accident occurs, the nearest emergency facility sends ambulances to it and injured people are conveyed to the nearest hospital. We propose a new multiobjective EFLP by extending Matsutomi and Ishii's EFLP.

EFLPs have the following two important factors. One is distance (or norm); for details of the relation between facility location and norm, the reader can refer to the study of Martini [6]. There are two norms widely used in studies about the EFLPs. One is the Euclidean norm [7, 8], which is assumed that it can be traveled to any orientations at any points. However, this assumption does not usually hold for facility location in urban areas. The other is the block norm [9-11], which is assumed that it can be traveled to given several allowable orientations of movement with weights at any points. Rectilinear distance [8] is regarded as one of the block norms such that there are two allowable orientations which cross at right angles with the same weights. The EFLPs with the rectilinear distance are often studied [7, 12]. The $A$-distance defined by Widmayer et al. [13] is also regarded as one of block norms such that there are several allowable orientations of movement with the same weights. Matsutomi and Ishii [2] consider an EFLP with the $A$ distance. In this paper, we propose a new EFLP based on the EFLP with the $A$-distance.

The other is criterion of optimality for facility location. In general EFLPs [2, 4, 7, 14, 15], an objective for facility location is to minimize the maximal distance between emergency facilities and the scenes of accidents. In this paper, we introduce another new objective, which is to maximize

*Address correspondence to this author at the Department of Artificial Complex Systems Engineering, Graduate School of Engineering, Hiroshima University, Higashihiroshima, Hiroshima, Japan;

E-mail: unotake@hiroshima-u.ac.jp frequency of accidents that emergency facilities can respond quickly. Then, a new multiobjective EFLP with the two objectives is formulated.

Most multiobjective EFLPs do not have complete optimal solutions. For finding a satisficing solution of the multiobjective EFLP for the decision maker (DM), we apply interactive fuzzy satisficing method proposed by Sakawa and Yano [16]. Katagiri et al. [17] recently proposed interactive fuzzy satisficing method for multiobjective fuzzy random linear programming problems. In this method, we need to find an optimal solution for each of the minimax problems with the corresponding reference membership values. Particle swarm optimization (PSO), which is proposed by Kennedy and Eberhart [18] and improved by Matsui et al. [19], is one of the efficient solution methods for nonlinear programming problem. We propose to apply the PSO improved by utilizing some characteristics of the EFLPs.

The organization of the paper is as follows. In Section 2, we give the definition of the $A$-distance and its properties. In Section 3, we formulate a multiobjective EFLP with the $A$ distance. For the formulated EFLP, first we propose the method to compute the objective values for each location in Section 4. In order to find a satisficing solution for the DM, we introduce the interactive fuzzy satisficing method proposed by Sakawa and Yano [16] in Section 5. In order to solve the minimax problems in this method, we proposed a PSO method considering characteristics of the EFLP in Section 6 . In section 7, we show the results for applying the method to numerical examples of the multiobjective EFLPs. Finally, we make mention of conclusions and future remarks in Section 8.

\section{$A$-DISTANCE}

In this section, we describe the definition of $A$-distance and its properties. We consider the situation that there are $a$ orientations which can only move in the plane $R^{2}$. The orientations are represented as the angles between the corresponding straight lines to orientations and the Cartesian $x$-axis; for example, orientation 0 is the $x$-axis and orientation $\pi / 2$ is 
the $y$-axis. Let $A=\left\{\alpha_{1}, \ldots, \alpha_{a}\right\}$ be a set of orientations such that $0 \leq \alpha_{1} \leq \cdots \leq \alpha_{a}<\pi$. A line, a half line, or a line segment is called $A$-oriented if its orientation is one of those in $A$. Then, the $A$-distance between two points $\boldsymbol{p}_{1}$ and $\boldsymbol{p}_{2} \in R^{2}$ is represented as follows:

$$
d_{A}\left(\mathbf{p}_{1}, \mathbf{p}_{2}\right):=\left\{\begin{array}{r}
d_{2}\left(\mathbf{p}_{1}, \mathbf{p}_{2}\right), \\
\text { if } \mathbf{p}_{1} \text { and } \mathbf{p}_{2} \text { are in } \\
\text { an } A \text {-oriented line, } \\
\min _{\mathbf{p}_{3} \in R^{2}}\left\{d_{A}\left(\mathbf{p}_{1}, \mathbf{p}_{3}\right)+d_{A}\left(\mathbf{p}_{3}, \mathbf{p}_{2}\right)\right\}, \\
\text { otherwise }
\end{array}\right.
$$

where $d_{2}(\cdot, \cdot)$ means the Euclidean distance. Fig. (1) shows an example of the A-distance between $\boldsymbol{p}_{1}$ and $\boldsymbol{p}_{2} \in R^{2}$. The rectilinear distance is represented as the $A$-distance with $A=\{0, \pi / 2\}$.

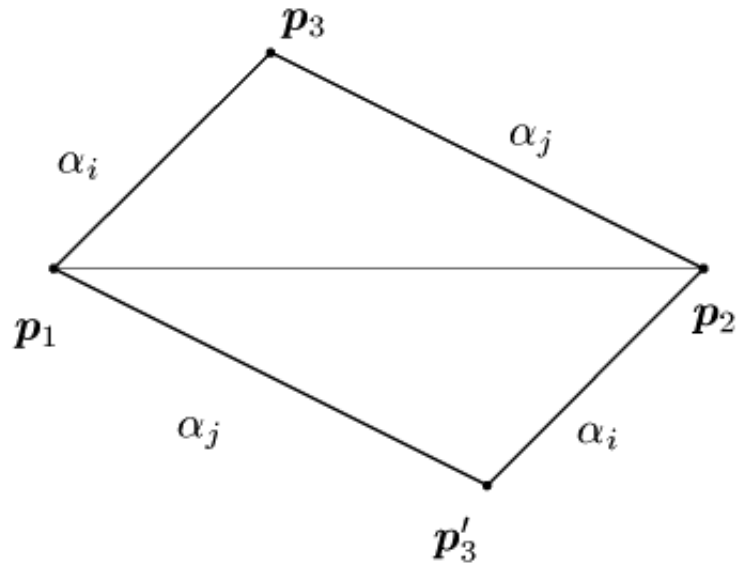

Fig. (1). An example of the $A$-distance.

Widmayer et al. [13] show that if points $\boldsymbol{p}_{1}$ and $\boldsymbol{p}_{2}$ are not in any $A$-oriented lines, there exists at least one point $p_{3} \in R^{2}$ such that

$d_{A}\left(\mathbf{p}_{1}, \mathbf{p}_{2}\right)=d_{2}\left(\mathbf{p}_{1}, \mathbf{p}_{3}\right)+d_{2}\left(\mathbf{p}_{3}, \mathbf{p}_{2}\right)$

For a point $\boldsymbol{p}$ and a distance $d>0$, the locus of all points $\boldsymbol{p}^{\prime}$ with $d_{A}\left(\boldsymbol{p}, \boldsymbol{p}^{\prime}\right)=d$ is called the $A$-circle with center $\boldsymbol{p}$ and radius $d$. As shown in Fig. (2), $A$-circle has its boundary of $2 a$-gon whose corner points are the intersections of the circle with center $\boldsymbol{p}$ and radius $d$ and the $A$-oriented lines through $\boldsymbol{p}$.

For two points $\boldsymbol{p}_{1}$ and $\boldsymbol{p}_{2}$, the bisector of $\boldsymbol{p}_{1}$ and $\boldsymbol{p}_{2}$ with the $A$-distance is defined as follows:

$B_{A}\left(\boldsymbol{p}_{1}, \boldsymbol{p}_{2}\right)=\left\{\boldsymbol{p} \mid d_{A}\left(\boldsymbol{p}, \boldsymbol{p}_{1}\right)=d_{A}\left(\boldsymbol{p}, \boldsymbol{p}_{2}\right)\right\}$

Let $Q=\left\{\boldsymbol{p}_{1}, \ldots, \boldsymbol{p}_{n}\right\}$ be a set of $n$ points $\boldsymbol{p}_{1}, \ldots, \boldsymbol{p}_{n} \in R^{2}$. Then, the Voronoi polygon $V_{A}\left(\boldsymbol{p}_{i}\right), i=1, \ldots, n$, with the $A$ distance is defined as follows:

$V_{A}\left(\mathbf{p}_{i}\right)=\bigcup_{j \neq i}\left\{\mathbf{p} \mid d_{A}\left(\mathbf{p}, \mathbf{p}_{i}\right) \leq d_{A}\left(\mathbf{p}, \mathbf{p}_{j}\right)\right\}$

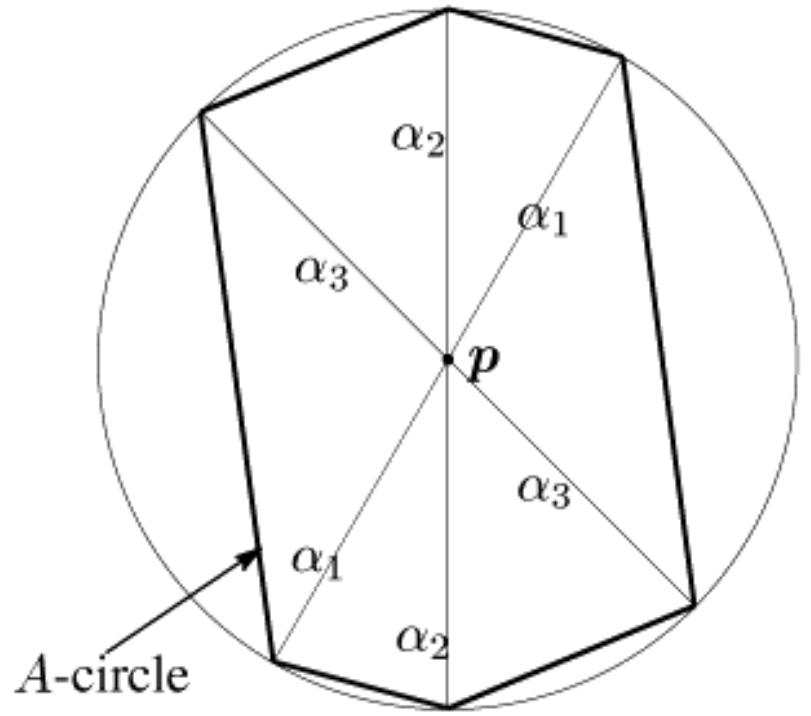

Fig. (2). An $A$-circle ( $A=\{\pi / 3, \pi / 2,3 \pi / 4\})$.

The sides and vertices of the Voronoi polygons are called Voronoi edges and Voronoi points, respectively. The set of all Voronoi polygons, which can be regarded as a partition of $R^{2}$, is called Voronoi diagram with the $A$-distance. Voronoi diagram for $Q$ is denoted by $V D_{A}(Q)$.

Theorem 1: A Voronoi diagram in the A-distance for $Q$ can be constructed in $O(n \log n)$ times using $O(n)$ space, which is asymptotically optimal in the worst case.

Proof: Correctness follows from the consideration in Section 3 of the reference [13]. Optimality is due, for instance, to a reduction of sorting [20].

Fig. (3) shows an example of the Voronoi diagram with $A=\{0, \pi / 2\}$.

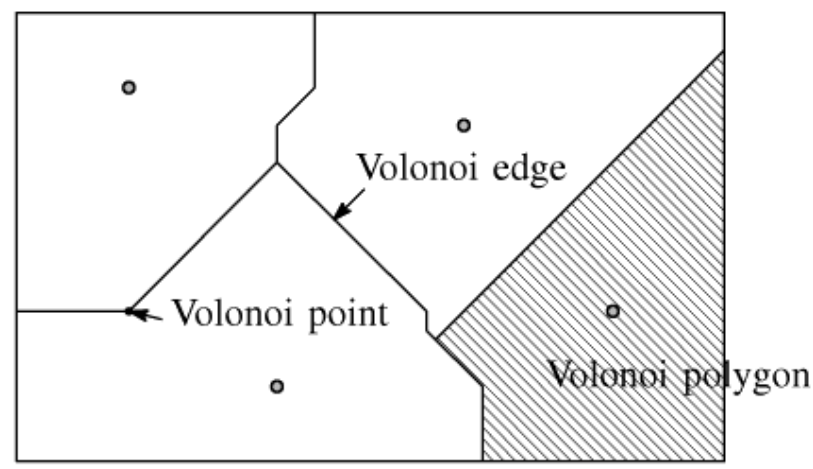

Fig. (3). Voronoi diagram with the $A$-distance.

\section{FORMULATION OF MULTI-OBJECTIVE EFLP}

In this section, we formulate a multiobjective EFLP with the $A$-distance. Let $S \subseteq R^{2}$ be a closed convex polygon in which accidents occur and the DM needs to locate emergency facilities. We consider the situation that if an accident occurs at a point, the nearest emergency facility to the point sends ambulances to the point and then injured people in the accident are conveyed from the point to the nearest hospital. 
First, we show the minimax criterion about path from the emergency facilities to the hospitals via the points of accidents. Let $\boldsymbol{h}_{1}, \ldots, \boldsymbol{h}_{m} \in S$ be sites of $m$ hospitals, let $\boldsymbol{y}_{1}, \ldots, \boldsymbol{y}_{n} \in S$ be sites of $n$ emergency facilities, and let $Y=\left(\boldsymbol{y}_{1}, \ldots, \boldsymbol{y}_{n}\right) \in S^{n}$. Then, if an accident occurs at a point $\boldsymbol{p} \in S$, the $A$-distance for the above path is represented as follows:

$u(Y, \mathbf{p})=\min _{i=1, \ldots, n} d_{A}\left(\mathbf{y}_{i}, \mathbf{p}\right)+\min _{j=1, \ldots, m} d_{A}\left(\mathbf{p}, \mathbf{h}_{j}\right)$

Because the DM does not know where accidents occur in $S$ beforehand, one of our objectives is interpreted as coping with any accident points in $S$ quickly. Then, the first objective function is represented as follows:

$$
f_{1}(Y):=\max _{\mathbf{p} \in S} u(Y, \mathbf{p})
$$

Secondly, we show a new criterion about frequency of accidents. We assume that the DM knows points where accidents frequently occur in $S$, called accident points. There are $k$ accident points whose sites are denoted by $\boldsymbol{a}_{1}, \ldots, \boldsymbol{a}_{k} \in S$, and each of their accident points has a weight about frequency of accidents, denoted by $w_{1}, \ldots, w_{k}>0$, respectively. Let $\gamma>0$ be an upper limit of the distance from the emergency facilities to the hospitals such that a medical treatment for injured people can be in time. The other of our objectives is interpreted as maximizing the sum of the weights of frequency of accident points that the emergency facilities can cover for a given $\gamma$. Then, the second objective function is represented as follows:

$$
f_{2}(Y):=\sum_{i \in I_{Y}(Y)} w_{i}
$$

where

$$
I_{\gamma}(Y):=\left\{\mathbf{a}_{i} \mid u(Y, \mathbf{p}) \leq \gamma\right\}
$$
lows:

Therefore, a multiobjective EFLP is formulated as fol-

$$
\left.\begin{array}{ll}
\text { minimize } & f_{1}(Y) \\
\text { maximize } & f_{2}(Y) \\
\text { subject to } & Y=\left(\mathbf{y}_{1}, \ldots, \mathbf{y}_{n}\right) \in S^{n}
\end{array}\right\}
$$

\section{COMPUTATION OF THE OBJECTIVE VALUES OF MULTIOBJECTIVE EFLP}

In order to find an optimal solution of (9), we need to compute the two objective values for each solution. For the second objective function, we can compute its objective value by measuring the $A$-distances from the emergency facilities to the hospitals via the $k$ accidents points. In the following part of this section, we propose the method to compute the first objective value for each location.

For the case $n=1$, Matsutomi and Ishii [2] showed the following theorem.

Theorem 2: If $n=1, \boldsymbol{p} \in S$ which maximizes $u(Y, \boldsymbol{p})$ is one of the following points:
- Vertices of the boundary of $S$,

- Intersections of Voronoi edges of each $V_{A}\left(\boldsymbol{h}_{1}\right), \ldots, V_{A}\left(\boldsymbol{h}_{m}\right)$ and the boundary of $S$.

If the DM locates two or more emergency facilities, we need to consider which of the emergency facilities is used at any points in $S$. From the definition of Voronoi polygon, $V_{A}\left(\boldsymbol{y}_{i}\right)$ is the set of points which uses emergency facility $i$. While $\boldsymbol{p} \in S$ which maximizes $u(Y, \boldsymbol{p})$ is only on the boundary of $S$ if $n=1, \boldsymbol{p} \in S$ which maximizes $u(Y, \boldsymbol{p})$ may be in the interior of $S$ and on the Voronoi edges of $V_{A}\left(\boldsymbol{h}_{1}\right), \ldots, V_{A}\left(\boldsymbol{h}_{m}\right)$ and $V_{A}\left(\boldsymbol{y}_{1}\right), \ldots, V_{A}\left(\boldsymbol{y}_{m}\right)$ if $n \geq 2$. Then, Theorem 1 can be extended to the following corollary:

Corollary 1: If $n \geq 2, \boldsymbol{p} \in S$ which maximizes $u(Y, \boldsymbol{p})$ is one of the following points:

- $\quad$ Vertices of the boundary of $S$,

- Intersections of Voronoi edges of each $V_{A}\left(\boldsymbol{h}_{1}\right), \ldots, V_{A}\left(\boldsymbol{h}_{m}\right)$ and the boundary of $S$,

- Voronoi points of each $V_{A}\left(\boldsymbol{h}_{1}\right), \ldots, V_{A}\left(\boldsymbol{h}_{m}\right)$,

- $\quad$ Voronoi points of each $V_{A}\left(\boldsymbol{y}_{1}\right), \ldots, V_{A}\left(\boldsymbol{y}_{m}\right)$,

- Intersections of Voronoi edges of each $V_{A}\left(\boldsymbol{y}_{1}\right), \ldots, V_{A}\left(\boldsymbol{y}_{m}\right)$ and the boundary of $S$,

- Intersections of Voronoi edges of each $V_{A}\left(\boldsymbol{h}_{1}\right), \ldots, V_{A}\left(\boldsymbol{h}_{m}\right)$ and Voronoi edges of each $V_{A}\left(\boldsymbol{y}_{1}\right), \ldots, V_{A}\left(\boldsymbol{y}_{m}\right)$.

The above points can be found by drawing Voronoi diagram for hospitals and Voronoi diagram for each location of emergency facilities. Then, we can find the first objective value by computing the maximal distance for paths from emergency facilities to hospitals via these points.

\section{INTERACTIVE FUZZY SATISFICING APPROACH}

In this section, we introduce the interactive fuzzy satisficing method proposed by Sakawa and Yano [16] in order to find a satisficing solution of (9) for the DM.

For decision making in real world, the DM usually prefer to make an objective function value more/less than a certain value rather than to maximize/minimize its objective function value. Such an objective, called "a fuzzy objective", includes vagueness based upon judgment of the DM. In this paper, we represent the two objectives of (9) as fuzzy objectives provided by membership functions, denoted by $\mu_{1}$ and $\mu_{2}$.

Now we introduce an example of membership functions for each objective function.

Let $d_{e}$ denote the distance such that the DM is quite satisfied if the first objective value is less than $d_{e}$, and $d_{l}$ denote the distance such that she/he is satisfied to a certain degree if its objective value is more than $d_{e}$ but less than 
$d_{l}$. Then, we use the following linear membership function for the former objective:

$$
\mu_{1}\left(f_{1}(Y)\right):= \begin{cases}1, & \text { if } f_{1}(Y)<d_{e}, \\ \frac{f_{1}(Y)-d_{e}}{d_{l}-d_{e}}, & \text { if } d_{e} \leq f_{1}(Y)<d_{l}, \\ 0, & \text { if } d_{l} \leq f_{1}(Y)\end{cases}
$$

Next, one of the simplest ways to provide membership function for the latter objective is as follows:

$$
\mu_{2}\left(f_{2}(Y)\right):=\frac{f_{2}(Y)}{\sum_{i=1}^{k} w_{i}}
$$

Then, (9) is transformed as the following multiobjective fuzzy programming problem:

$$
\left.\begin{array}{ll}
\operatorname{minimize} & \mu_{1}\left(f_{1}(Y)\right) \\
\text { maximize } & \mu_{2}\left(f_{2}(Y)\right) \\
\text { subject to } & Y=\left(\mathbf{y}_{1}, \ldots, \mathbf{y}_{n}\right) \in S^{n}
\end{array}\right\}
$$

Since there is generally no complete optimal solution for multiobjective programming problem including (12), the concept of the M-Pareto optimal solution is usually used for multiobjective fuzzy programming problems.

Definition 1: Solution $Y *$ is an M-Pareto optimal solution of (12) if and only if there does not exist any solutions $Y \in S^{n}$ such that $\mu_{i}\left(f_{i}(Y)\right) \geq \mu_{i}\left(f_{i}\left(Y^{*}\right)\right)$ for all $i=1,2$ and $\mu_{j}\left(f_{j}(Y)\right)>\mu_{j}\left(f_{j}\left(Y^{*}\right)\right)$ for at least one $j \in\{1,2\}$.

The interactive fuzzy satisficing method [16] is to find a satisficing M-Pareto optimal solution through interaction to the DM. Let $\left(\bar{\mu}_{1}, \bar{\mu}_{2}\right)$ be a pair of initial reference membership levels of membership function $\mu_{1}$ and $\mu_{2}$, respectively. The interactive fuzzy satisficing method for (12) can be described as follows:

\section{Algorithm 1: Interactive Fuzzy Satisficing Method}

Step 1. Provide two membership functions $\mu_{1}$ and $\mu_{2}$ according to (10) and (11).

Step 2. Set the initial reference membership levels $\left(\bar{\mu}_{1}, \bar{\mu}_{2}\right)=(1,1)$.

Step 3. For the given pair of reference membership levels $\left(\bar{\mu}_{1}, \bar{\mu}_{2}\right)$, solve the following corresponding minimax problem:

$$
\left.\begin{array}{cc}
\operatorname{minimize} & \max _{i=1,2}\left\{\bar{\mu}_{i}-\mu_{i}\left(f_{i}(Y)\right)\right. \\
& \left.+\rho \sum_{j=1}^{2}\left(\bar{\mu}_{i}-\mu_{i}\left(f_{i}(Y)\right)\right)\right\} \\
\text { subject to } & Y=\left(\mathbf{y}_{1}, \ldots, \mathbf{y}_{n}\right) \in S^{n}
\end{array}\right\}
$$

where, $\rho$ is a sufficiently small positive number.

Step 1. If the DM is satisfied with the current levels of the M-Pareto optimal solution, STOP. Then the cur- rent M-Pareto optimal solution is a satisficing solution for the DM.

Step 2. Update the pair of current reference membership levels $\left(\bar{\mu}_{1}, \bar{\mu}_{2}\right)$ based on information of preference of the DM, the current values of the membership functions, etc. Return to Step 3.

In the interactive fuzzy satisficing method, we need to solve the minimax problems in Step 3 efficiently. In the next section, we propose an efficient solution method for the minimax problem.

\section{A SOLUTION ALGORITHM FOR MINIMAX PROB- LEM}

A PSO method proposed by Kennedy and Eberhart [18] is based on the social behavior that a population of individuals adapts to its environment by returning to promising regions that were previously discovered [21]. This adaptation to the environment is a stochastic process that depends upon both the memory of each individual, called particle, and the knowledge gained by the population, called swarm.

In the numerical implementation of this simplified social model, each particle has the following three attributes: the position vector in the search space, the velocity vector and the best position in its track, and the best position of the swarm. The process can be outlined as follows.

\section{Algorithm 2: Outline of the PSO Method}

Step 1. Generate the initial swarm involving $N$ particles at random.

Step 2. Calculate the new velocity vector for each particle, based on its attributes.

Step 3. Calculate the new position of each particle from the current position and its new velocity vector.

Step 4. If the terminal condition is satisfied, STOP. The best solution given in the searching history is an approximate optimal solution. Otherwise, go to Step 2.

To be more specific, for the position and the velocity vector of the $i$-th particle at time $t$, denoted by $\boldsymbol{x}_{i}^{t}$ and $\boldsymbol{v}_{i}^{t}$, respectively, the new velocity vector of the $i$-th particle at time $t+1$ is calculated by the following scheme introduced by Shi and Eberhart [22].

$$
\boldsymbol{v}_{i}^{t+1}:=\omega^{t} \boldsymbol{v}_{i}^{t}+c_{1} R_{1}^{t}\left(\boldsymbol{p}_{i}^{t}-\boldsymbol{x}_{i}^{t}\right)+c_{2} R_{2}^{t}\left(\boldsymbol{p}_{g}^{t}-\boldsymbol{x}_{i}^{t}\right)
$$

where $R_{1}^{t}$ and $R_{2}^{t}$ are random numbers between 0 and 1 at time $t, \boldsymbol{p}_{i}^{t}$ is the best position of the $i$-th particle in its track and $\boldsymbol{p}_{g}^{t}$ is the best position of the swarm. There are three problem-dependent parameters, the inertia of the particle $\omega^{t}$, and two trust parameters $c_{1}$ and $c_{2}$. Then, the new position of the $i$-th particle at time $t+1$ is calculated from the following equation:

$x_{i}^{t+1}:=x_{i}^{t}+v_{i}^{t+1}$ 
The $i$-th particle calculates the next search direction vector $\boldsymbol{v}_{i}^{t+1}$ by (14) in consideration of the current search direction vector $\boldsymbol{v}_{i}^{t}$, the direction vector going from the current search position $\boldsymbol{x}_{i}^{t}$ to the best position in its track $\boldsymbol{p}_{i}^{t}$ and the direction vector going from the current search position $\boldsymbol{x}_{i}^{t}$ to the best position of the swarm $\boldsymbol{p}_{g}^{t}$, moves from the current position $\boldsymbol{x}_{i}^{t}$ to the next search position $\boldsymbol{x}_{i}^{t+1}$ calculated by (14). The parameter $\omega^{t}$ controls the amount of the move to search globally in early stage and to search locally by decreasing $\omega^{t}$ gradually. It is defined by follows:

$\omega^{t}:=\omega^{0}-\frac{t\left(\omega^{0}-\omega^{T_{\max }}\right)}{0.75 T_{\max }}$

where $T_{\max }$ is the number of maximum iteration times, $\omega^{0}$ is an initial value at the time iteration, and $\omega^{T_{\max }}$ is the last value.

The searching procedure of PSO is shown in Fig. (4).

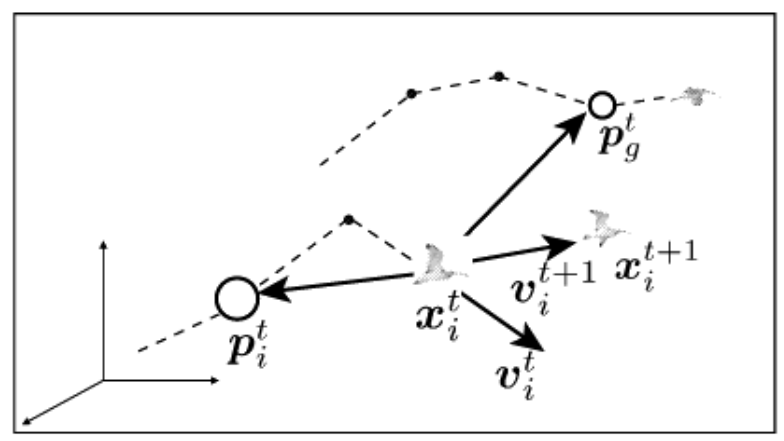

Fig. (4). Movement model for PSO.

Comparing the evaluation value of a particle after movement, denoted by $f\left(\boldsymbol{x}_{i}^{t+1}\right)$, with that of the best position in its track, denoted by $f\left(\boldsymbol{p}_{i}^{t}\right)$, if $f\left(\boldsymbol{x}_{i}^{t+1}\right)$ is better than $f\left(\boldsymbol{p}_{i}^{t}\right)$, then the best position in its track is updated as $f\left(\boldsymbol{p}_{i}^{t+1}\right) \leftarrow f\left(\boldsymbol{x}_{i}^{t+1}\right)$. Otherwise, then the best position in the swarm is updated as $f\left(\boldsymbol{p}_{i}^{t+1}\right) \leftarrow f\left(\boldsymbol{p}_{i}^{t}\right)$. In this way, a particle gets information of the best position of new oneself and the swarm, and moves according to (14) and (15) again, and searches newly. The summary of the PSO method is shown in Fig. (5).

Such a PSO technique includes two drawbacks. One is that particles concentrate on the best search position of the swarm and they cannot easily escape from the local optimal solution since the move direction vector $\boldsymbol{v}_{i}^{t+1}$ calculated by (14) always includes the direction vector to the best search position of the swarm. Another is that a particle after move is not always feasible for problems with constraints.

In order to settle the first issue, Matsui et al. [19] proposed "the leaving acts" for particles which are on the best position of the swarm. Moreover, they proposed the multiplex stretching method, which is the extended version of the stretching method proposed by Parsopoulos and Varahatis [23]. In order to settle the second problem, Matsui et al. [19] proposed to generate initial particles in the feasible set by utilizing the homomorphism proposed by Koziel and Michalewicz [24]. Moreover, there are often cases that a particle after move is not always infeasible if we use the updating equation of search position mentioned above. To deal with such a situation, Matsui et al. [19] divided the swarm into two subswarms; one is the move of a particle to the infeasible region is accepted and the other is not.

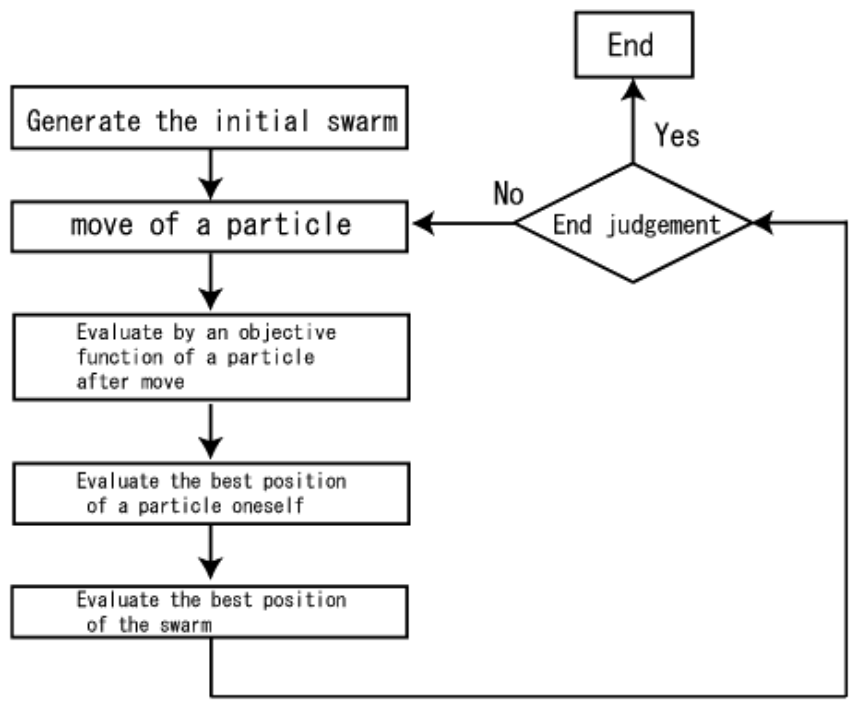

Fig. (5). Summary of PSO algorithm.

\section{IMPROVEMENT OF PSO METHOD}

In this study, based upon the PSO method introduced in the previous section, we proposed to add a new fourth movement in (14). Let $\boldsymbol{q} \in S$ be maximizer of $u\left(\boldsymbol{x}_{i}^{t}, \boldsymbol{p}\right)$, such a point can be found by using Theorem 1. In (9) approaching the nearest emergency facility to $\boldsymbol{q}$, objective value of $f_{1}(Y)$ may be improved. Therefore we introduce such a movement to our solution method. Let $\boldsymbol{p}_{a}^{t}$ be a position such that for $\boldsymbol{x}_{i}^{t}$, a site of the nearest facility is changed to $\boldsymbol{q}$ and sites of the other facilities are fixed. Then, our proposing new velocity vector of the $i$-th particle at time $t+1$ is represented as follows:

$$
\begin{aligned}
\mathbf{v}_{i}^{t+1}:=\omega^{t} \mathbf{v}_{i}^{t}+c_{1} R_{1}^{t}\left(\mathbf{p}_{i}^{t}-\mathbf{x}_{i}^{t}\right) & +c_{2} R_{2}^{t}\left(\mathbf{p}_{g}^{t}-\mathbf{x}_{i}^{t}\right) \\
& +c_{3} R_{3}^{t}\left(\mathbf{p}_{a}^{t}-\mathbf{x}_{i}^{t}\right)
\end{aligned}
$$

where $c_{3}$ is a trust parameter and $R_{3}^{t}$ is a random number between 0 and 1 .

\section{NUMERICAL EXPERIMENTS}

In this section, we apply interactive fuzzy satisficing approach with the PSO method to an example of our proposing multiobjective EFLPs. In this example, we consider an EFLP 
for two emergency facilities, that is $n=2$. We represent $S$ as a convex hull including 20 points given in $[0,100] \times[0,100]$ randomly. For the $A$-distance, we set $A=\{0, \pi / 4, \pi / 2,3 \pi / 4\}$. For hospitals, we set $m=3$ and their sites are given in $S$ randomly. For the frequency of accidents, we set $\gamma=15$, and for each of 100 accident points, its site and weight are randomly given in $S$ and $(0,1]$, respectively.

We illustrate the interactive fuzzy satisficing approach for the above example of the multiobjective EFLP. For parameters of PSO, we use the same parameter as the study of Matsui et al. [19], that is, its population size is 40, its generation is 500 , and $c_{1}=c_{2}=2$. Moreover, we set the new parameter $c_{3}=0.5$.

At Step 1, in order to represent fuzziness about two objectives, we use membership functions (10) and (11) in Section 5 with setting $d_{e}=5$ and $d_{l}=120$.

At Step 3, we solve minimax problem (13) for each given $\left(\bar{\mu}_{1}, \bar{\mu}_{2}\right)$ by solving the PSO method in section 6 . We set $\rho=10^{-3}$. In order to verify efficiency of PSO method, we apply the solution method GENOCOP [24], which is a genetic algorithm for numerical optimization for constrained problem proposed by Koziel and Michalewicz [24], to minimax problems. For parameters of GENOCOP, we set the same population size and generation. Moreover, we set the other parameters similarly to the study of Koziel and Michalewicz [24]. Computational results for each given $\left(\bar{\mu}_{1}, \bar{\mu}_{2}\right)$ at 20 times by PSO and GENOCOP are shown in Tables 1 and $\mathbf{2}$, respectively, where these results are given by using DELL Optiplex GX620 (CPU: Pentium(R) 4 2.33GHz, RAM: 512MB).

Table 1. Computational Results by PSO

\begin{tabular}{|c|c|c|c|}
\hline Minimax Problem & $\mathbf{1}$ & $\mathbf{2}$ & $\mathbf{3}$ \\
\hline \hline $\bar{\mu}_{1}$ & 1.0 & 1.0 & 0.9 \\
\hline $\bar{\mu}_{2}$ & 1.0 & 0.8 & 0.8 \\
\hline Best & 0.4104 & 0.3109 & 0.2608 \\
\hline Mean & 0.4106 & 0.3113 & 0.2611 \\
\hline Worst & 0.4113 & 0.3120 & 0.2614 \\
\hline Mean CPU & 9.5196 & 10.080 & 9.0718 \\
\hline Time (Sec) & & & \\
\hline
\end{tabular}

From Tables 1 and 2, PSO can find better solutions than GENOCOP by meanings of both mean and stability. This means that efficiency of PSO for such minimax problems.

At Step 4, the DM evaluates whether the M-Pareto optimal solution given by solving the minimax problem at Step 3 is satisfied or not. If its solution satisfies the DM, this algorithm is terminated. Otherwise, ask the DM to update the
Table 2. Computational Results by GENOCOP

\begin{tabular}{|c|c|c|c|}
\hline Minimax Problem & $\mathbf{1}$ & $\mathbf{2}$ & $\mathbf{3}$ \\
\hline \hline $\bar{\mu}_{1}$ & 1.0 & 1.0 & 0.9 \\
\hline $\bar{\mu}_{2}$ & 1.0 & 0.8 & 0.8 \\
\hline Best & 0.4132 & 0.3111 & 0.2611 \\
\hline Mean & 0.4302 & 0.3292 & 0.2764 \\
\hline Worst & 0.4483 & 0.3440 & 0.2885 \\
\hline $\begin{array}{c}\text { Mean CPU } \\
\text { Time (Sec) }\end{array}$ & 10.101 & 11.982 & 10.044 \\
\hline
\end{tabular}

current reference membership levels $\left(\bar{\mu}_{1}, \bar{\mu}_{2}\right)$ by considering the current values of the membership functions, and resolve minimax problem to Step 3. In this example of EFLP, we assume that the DM thinks that the first objective is more important than the second objective. Then the DM hopes to improve the value of $\mu_{1}$ even if the value of $\mu_{2}$ is changed for the worse. However, the DM does not hope to go the value of $\mu_{2}$ too bad. Then, an example of the interactive fuzzy satisficing methods is shown in Table $\mathbf{3}$.

Table 3. Results of Interactive Fuzzy Satisficing Approach

\begin{tabular}{|c|c|c|c|}
\hline Iteration & $\mathbf{1}$ & $\mathbf{2}$ & $\mathbf{3}$ \\
\hline \hline $\bar{\mu}_{1}$ & 1.0 & 1.0 & 0.9 \\
\hline $\bar{\mu}_{2}$ & 1.0 & 0.8 & 0.8 \\
\hline$\mu_{1}\left(f_{1}\left(Y^{*}\right)\right)$ & 0.5901 & 0.6892 & 0.6395 \\
\hline$\mu_{2}\left(f_{2}\left(Y^{*}\right)\right)$ & 0.5897 & 0.4892 & 0.5393 \\
\hline
\end{tabular}

In Table 3, the DM considers as follows:

- $\quad$ At Iteration 1, the DM is not satisfied M-Pareto optimal solution because the first objective value is bad.

- At Iteration 2, for improving the first objective value, the DM decreases $\bar{\mu}_{2}$, which is reference membership levels for the second objective. Then, the MPareto optimal solution given at Iteration 2 is improved for the first objective. However, she/he is not satisfied because the second objective value is too bad.

- At Iteration 3, for improving the second objective value a little, the DM decreases $\bar{\mu}_{1}$, which is reference membership levels for the first objective. Then, she/he obtains a satisficing solution for both two objectives, so the algorithm is terminated.

\section{CONCLUSIONS AND FUTURE STUDIES}

In this paper, we have proposed a new multiobjective EFLP with the $A$-distance. In order to obtain a satisficing 
solution for the DM, we have proposed an interactive fuzzy satisficing method which involves the procedure of solving minimax problems by the PSO method. By applying an example of multiobjective EFLPs, we showed efficiency of PSO and illustrated the interactive fuzzy satisficing method.

In the multiobjective EFLPs, we assume that the region of facility location $S$ is a convex polygon because Theorem 2 and Corollary 1 use the assumption. However, in order to apply the EFLPs to more general cases, we need to consider various shapes of $S$ which are non-convex, non-connected, etc. To construct our solution method for general shapes of $S$ is a future study. Moreover, if $S$ includes many hospitals and the DM locates many emergency facilities in $S$, we need to find an optimal solution more efficiently for the minimax problems in the interactive fuzzy satisficing methods. To consider an efficient solution method for large-scale multiobjective EFLPs is also a future study.

\section{REFERENCES}

[1] C. Araz, H. Selim, and I. Ozkarahan, "A fuzzy multi-objective covering-based vehicle location model for emergency services", Comput. Operat. Res., vol. 34, no. 3, pp. 705-726, March 2007.

[2] T. Matsutomi and H. Ishii, "Minimax location problem with $A$ distance”, J. Opl. Res. Soc., vol. 41, pp. 181-195, June 1998.

[3] H. K. Rajagopalan, C. Saydam, and J. Xiao, "A multiperiod set covering location model for dynamic redeployment of ambulances", Comput. Operat. Res., vol. 35, no. 3, pp. 814-826, March 2008.

[4] D. R. Plane and T. E. Hendric, "Mathematical programming and the location of fire companies for the Denver fire department", Opns. Res., vol. 25, pp. 563-578, July-August 1977.

[5] L. Yang, B. F. Jones, and S. H. Yang, "A fuzzy multi-objective programming for optimization of fire station locations through genetic algorithms", Eur. J. Oper. Res., vol. 181, no. 2, pp. 903-915, September 2007.

[6] H. Martini and A. Schöbel, "Median hyperplanes in normed spaces - a survey”, Discrete Appl. Math., vol. 89, pp. 181-195, December 1998.

[7] J. Elzinga and D. W. Hearn, "Geometrical solutions for some minimax location problems", Trans. Sci., vol. 6, pp. 379-394, November 1972

[8] S. Özşen and S. Güneş, "Effect of feature-type in selecting distance measure for an artificial immune system as a pattern recognizer" Digital Signal Processing (In Press.)

[9] B. Pelegrin and F. R. Fernandez, "Determination of efficient points in multiple-objective location problems", Navel Res. Log., vol. 35, pp. 697-705, December 1988.
[10] J. F. Thisse, J. E. Hendric, and R. E. Wendell, "Some properties of location problems with block and round norm", Opns. Res., vol. 32, pp. 1309-1327, November-December 1984.

[11] J. E. Ward and R. E. Wendell, "Using block norm for location modeling”, Opns. Res., vol. 33, pp. 1074-1090, September-October 1985.

[12] G. O. Wesolowsky, "Rectangular distance location under the minimax optimality criterion", Trans. Sci., vol. 6, pp. 103-113, February 1972.

[13] P. Widmayer, Y. F. Wu, and C. K. Wong, "On some distance problems in fixed orientations", SIAM J. Comput., vol. 16, pp. 728-746, August 1987.

[14] R. L. Francis, "A geometrical solution procedure for a rectilinear minimax location problem”, AIIE Trans., vol. 4, pp. 328-332, December 1972.

[15] D. K. Kulshrestha, "A mini-max location problem with demand points arbitrarily distributed in a compact connected space", J. Opl. Res. Soc., vol. 38, pp. 447-452, May 1987.

[16] M. Sakawa and H. Yano, "An interactive fuzzy satisficing method using augmented minimax problems and its application to environmental systems", IEEE Trans. Syst. Man Cybern., vol. SMC-15, pp. 720-729, November-December 1985.

[17] H. Katagiri, M. Sakawa, K. Kato, and I. Nishizaki, "Interactive multiobjective fuzzy random linear programming: Maximization of possibility and probability", Eur. J. Oper. Res., (In Press.)

[18] J. Kennedy and R. C. Eberhart, "Particle swarm optimization", in Proc. of IEEE Int. Conf. Neural Networks, Piscataway, NJ, pp. 1942-1948, 1995.

[19] T. Matsui, M. Sakawa, T. Uno, K. Kato, M. Higashimori, and M. Kaneko, "Particle swarm optimization for jump height maximization of a serial link robot", Journal of Advanced Computational Intelligence and Intelligent Informatics, vol. 11, no. 8, pp. 956-963, Octorber 2007.

[20] F. Hwang, "An $O(n \log n)$ algorithm for rectilinear minimal spanning trees", J. Assoc. Comput. Mach., vol. 26, pp. 177-182, April 1979.

[21] J. Kennedy, W. M. Spears, "Matching algorithms to problems: an experimental test of the particle swarm and some genetic algorithms on the multimodal problem generator", in Proc. of IEEE Int. Conf. Evolutionary Computation, Anchorage, Alaska, 1998.

[22] Y. Shi and R. C. Eberhart, "A modified particle swarm optimizer", in Proc. of IEEE Int. Conf. Evolutionary Computation, Anchorage, Alaska, 1998.

[23] K. E. Parsopoulos and M. N. Varahatis, "Recent approaches to global optimization problems through Particle Swarm Optimization", Nat. Comput., vol. 1, pp. 235-306, June 2002.

[24] S. Koziel and Z. Michalewicz, "Evolutionary algorithms, homomorphous mappings, and constrained parameter optimization", Evol. Comput., vol. 7, no. 1, pp. 19-44, Spring 1999. 